\title{
Impact of the COVID-19 pandemic on surgery for severe endometriosis in the UK: a national database study
}

\author{
Jonathan Lewin ${ }^{1}$, Ertan Saridogan ${ }^{2}$, Dominic Byrne ${ }^{3}$, T Justin Clark ${ }^{4}$, and Arvind \\ Vashisht $^{5}$ \\ ${ }^{1}$ University College London \\ ${ }^{2}$ University College London Hospitals NHS Foundation Trust \\ ${ }^{3}$ Royal Cornwall Hospitals NHS Trust \\ ${ }^{4}$ Birmingham Women's NHS Foundation Trust \\ ${ }^{5}$ University College London Hospitals NHS Foundation Trust Women's Health Service
}

September 25, 2021

\begin{abstract}
Objective: To determine the impact of the COVID-19 pandemic on surgery for severe endometriosis in the UK at a national, regional and centre-level. Design: Population-based national cohort study. Population: All women undergoing endometriosis surgery requiring dissection of the pararectal space in the UK from 2017 to 2020 inclusive. Methods: The British Society for Gynaecological Endoscopy (BSGE) collects data nationally on all operations for severe endometriosis which involve dissection of the pararectal space. Annual audits of this database were obtained from the BSGE. Publicly available data on COVID-19 deaths and population were obtained from the UK Office for National Statistics. Main outcome measures: Numbers of annual BSGE-registered endometriosis operations. Results: A total of 5916 operations were performed. The number of operations decreased by $49.4 \%$ overall between 2019 and 2020. The number of endometriosis centres remained the same, however the median number of operations per centre decreased from 21 to 12.5, with a median percentage decrease at each centre of $51.0 \%$ (IQR 29.4\% - 75.0\%). There was no significant change in the type of surgery performed. All 11 administrative regions of Great Britain had reduced numbers of BSGE-registered operations in 2020 compared with the average for 2017-2019, with a median $56.6 \%$ decrease (range $6.13 \%-68.62 \%$ ). Regional reduction in operations was significantly correlated with COVID-19 death rates $(\mathrm{r}=0.557,95 \% \mathrm{CI}$ of $\mathrm{r} 0.048-1.00, \mathrm{p}=0.037)$. Conclusions: There has been a dramatic fall in the number of operations for severe endometriosis in Britain during the COVID-19 pandemic.
\end{abstract}

Authors: Dr Jonathan Lewin, University College London Hospital, Department of Gynaecology. 235 Euston Road London NW1 2BU Professor Ertan Saridogan, University College London Hospital, Department of Gynaecology. 235 Euston Road London NW1 2BU Mr Dominic Byrne, Royal Cornwall Hospital, Department of Gynaecology. Treliske, Truro TR1 3LJ Professor Thomas Justin Clark, Birmingham Women's and Children's Hospital, Department of Gynaecology. Mindelsohn Way, Birmingham B15 2TG Mr Arvind Vashisht, University College London Hospital, Department of Gynaecology. 235 Euston Road London NW1 $2 \mathrm{BU}$

Corresponding Author: Dr Jonathan Lewin. Contact Address: Flat F, 14 John Spencer Square, London N1 2LZ. Tel: +447968842291. Email: Jonathan.lewin1@nhs.net

Running Title: Impact of COVID-19 on endometriosis surgery in the UK

Full Abstract: Objective: To determine the impact of the COVID-19 pandemic on surgery for severe endometriosis in the UK at a national, regional and centre-level. 
Design: Population-based national cohort study. Population: All women undergoing endometriosis surgery requiring dissection of the pararectal space in the UK from 2017 to 2020 inclusive. Methods: The British Society for Gynaecological Endoscopy (BSGE) collects data nationally on all operations for severe endometriosis which involve dissection of the pararectal space. Annual audits of this database were obtained from the BSGE. Publicly available data on COVID-19 deaths and population were obtained from the UK Office for National Statistics. Main outcome measures: Numbers of annual BSGE-registered endometriosis operations. Results: A total of 5916 operations were performed. The number of operations decreased by $49.4 \%$ overall between 2019 and 2020. The number of endometriosis centres remained the same, however the median number of operations per centre decreased from 21 to 12.5, with a median percentage decrease at each centre of $51.0 \%$ (IQR $29.4 \%-75.0 \%$ ). There was no significant change in the type of surgery performed. All 11 administrative regions of Great Britain had reduced numbers of BSGE-registered operations in 2020 compared with the average for 2017-2019, with a median $56.6 \%$ decrease (range $6.13 \%-68.62 \%$ ). Regional reduction in operations was significantly correlated with COVID-19 death rates ( $\mathrm{r}=0.557,95 \%$ CI of r $0.048-1.00, \mathrm{p}=0.037)$. Conclusions: There has been a dramatic fall in the number of operations for severe endometriosis in Britain during the COVID-19 pandemic. Funding: None Keywords: Endometriosis, COVID-19, Pandemic, BSGE

Tweetable Abstract: The number of operations for severe endometriosis in Britain fell by $49 \%$ in 2020 vs pre-pandemic levels.

\section{Introduction}

The COVID-19 pandemic has had a significant impact on surgical services in the UK, with more than 1.5 million operations cancelled or postponed in 2020. In order to increase ITU capacity to care for ventilated patients, operating theatres throughout the country were transformed into high-dependency units. Anaesthetists required for elective surgery were unavailable as their unique skills in caring for the critically ill were needed for COVID patients. Many other non-emergency teams and healthcare professionals were redeployed to help as frontline workers to combat the pandemic and treat afflicted patients. Many other staff have been unable to work in operating theatres due to illness or shielding for pre-existing health conditions. Patients have faced reduced access to general practitioner (GP) services and specialist surgical clinics, all of which have prevented or delayed the scheduling of operations.

Prior to the pandemic patients already faced long waits for elective surgery, and this is particularly true of endometriosis surgery. Endometriosis patients in the UK wait on average 8 years for diagnosis alone, and $30 \%$ wait more than 10 months for surgery. The delay in surgery incurs secondary economic costs such as time out of education and employment, and places additional strain on emergency services from attendances for uncontrolled pain. It is vital that the backlog of endometriosis surgery is quantified, and a plan is made to reduce the waiting times for endometriosis surgery to a sustainable level. The pandemic will undoubtedly have adversely impacted this position, although the distribution and scale of the interruption to endometriosis surgery is not yet known.

The British Society for Gynaecological Endoscopy (BSGE) is an organisation set up by clinicians to improve standards, training and exchange of information in gynaecological minimal access surgery, and is the primary national aggregator of data on endometriosis surgery in the UK. To gain accreditation as a BSGE-approved endometriosis centre, a unit must provide data on complex operations to a centralised database which is audited annually to maintain accreditation. This database provides a register of the state of surgical services for severe endometriosis in the UK, and therefore has the potential to help assess the impact of the COVID19 pandemic on these services. The purpose of this study is to assess the impact of COVID-19 on surgery for severe endometriosis at a national, regional and centre-level using the BSGE database, and to assess the correlation between pandemic severity and disruption to these services. 


\section{Methods}

To be granted BSGE annual accreditation as an endometriosis centre, criteria must be met including a minimum number of complex surgical cases, multidisciplinary team involvement and submission of an exemplar surgical video. Auditing is performed annually and case numbers per surgeon must be adequate for each centre to maintain its accreditation each year. At least 12 cases of laparoscopic surgical excision of rectovaginal endometriosis requiring dissection of the pararectal space per surgeon are required, and any such case must be registered on the national BSGE database.

Annual audits of the BSGE database were obtained from the BSGE endometriosis centres committee including data from all accredited centres from 2017 - 2020 inclusive to analyse the impact of the COVID-19 pandemic. Data included the number of operations performed annually at each centre and the type of bowel surgery performed. Region-level data on operations were aggregated according to the region of the centre at which the operations were performed. Centres from outside the UK were excluded, as well as Northern Ireland due to lack of data.

Data on regional COVID deaths were obtained from publicly available data compiled by the UK Office of National Statistics (ONS) and the National Registry of Scotland. Regional population estimates were obtained from the ONS and used to calculate registered COVID deaths per 1000 population. Statistical analysis was performed using Pearson $\mathrm{r}$ correlation coefficient and $\mathrm{Chi}^{2}$ goodness-of-fit test in $\mathrm{R}$ with the Base package. Graphs were plotted in $\mathrm{R}$ using RStudio with the GGPLOT2 package, and regional mapping templates were obtained from the ONS.

\section{Results}

\section{Centres}

A total of 73 centres were registered with the BSGE between 2017 to 2020 . Over this time period, the number of centres submitting data for registered operations each year increased from 56 to 66 (Figure 1). Four centres submitted registered operations for the first time in 2020, while seven previously registered centres did not register any operations in 2020. Out of 73 centres, 48 submitted registered operations every year, 13 only for 3 years, 8 only for 2 years and 4 only for 1 year.

\section{Operations}

Over the time period from 2017 - 2020, a total of 5916 operations were performed for deep endometriosis requiring pararectal dissection at BSGE-accredited centres (Figure 2). The total number of registered operations increased by an average of $13.5 \%$ per year between $2017-2019$, then decreased by $49.4 \%$ between 2019-2020.

At a centre-level, 60 endometriosis centres had reduced operation numbers in 2020 compared with the average for 2017-2019, while 9 centres had increased numbers of operations. Overall, the annual median number of operations per centre decreased from 21 operations to 12.5 operations, with a median decrease in operations per centre of $51.0 \%$ (IQR 29.4\% - 75.0\%).

Between 2017 and 2019, on average $33.0 \%$ of patients had no surgery on the bowel, $60.2 \%$ underwent shaving of endometriosis from the bowel surface, $1.8 \%$ underwent a disc resection and $5.0 \%$ underwent resection of a segment of bowel. In 2020,34.5\%, 59.3\%, $2.6 \%$ and $3.6 \%$ of patients underwent no bowel surgery, bowel shave, disc resection and segmental bowel resection respectively, which was not significantly different to previous years $(\mathrm{p}=0.08)$. 


\section{Regions}

At a regional level, all 11 administrative regions of Great Britain had reduced numbers of BSGE-registered operations for endometriosis in 2020 compared with averages for 2017-2019, with a median $56.6 \%$ decrease (range 6.13\% - 68.62\%) (Figures, 3 Figure 4). Regions had a median of 1.49 deaths from COVID-19 per 1000 population (range $0.80-1.91$ ). At a regional level, reduction in these operations was significantly correlated with COVID-19 death rates ( $\mathrm{r}=0.56,95 \%$ CI of r $0.048-1.00, \mathrm{p}=0.037)$.

\section{Discussion}

\section{Main Findings}

Our study demonstrates the dramatic reduction in endometriosis surgery by nearly $50 \%$ overall, . This is significantly higher than the $38.6 \%$ reduction in elective procedures nationwide, indicating that endometriosis surgery has been particularly badly affected. the number of endometriosis operations per centre decreased by a median $51 \%$, while the number of centres remained approximately the same. Only 9 centres had increased operation numbers, which may have been newer centres in the process of growing their workload as usually occurs when centres acquire additional staff. Despite these changes, the proportions of patients undergoing the different types of surgery on the bowel (shaving, disc resection and segmental resection) remains unaffected. Reduction in endometriosis operations at a regional level were correlated with regional COVID-19 death rates.

\section{Strengths and Limitations}

This study is the first to provide a comprehensive assessment of the effect of the COVID-19 pandemic on surgery for severe endometriosis in the UK. A nation-wide, standardised approach was taken in data collection, however only BSGE-registered operations were taken into account, and there may have been other operations performed which were not registered with the BSGE. There was also a lack of data from Northern Ireland, restricting analysis to Great Britain.

\section{Interpretation}

The magnitude of the reduction in operations for this common, chronic, unremitting gynaecological condition is of major concern, and highlights the challenges for the restoration of endometriosis services. There is a pressing need in the pandemic recovery period for substantial investment to provide the necessary resources for adequate surgical service provision. Despite national prioritisation guidance stating that surgery for severe endometriosis with uncontrolled pain should be performed within 3 months, it is likely that patients will experience significantly longer wating times than this, as they did prior to the pandemic. Indeed, NHS England statistics from March 2021 show only $65.3 \%$ of patients receive treatment within 18 weeks of referral for gynaecological disease, and this figure may be considerably worse for endometriosis treatment.

Most endometriosis surgery is performed laparoscopically, and part of the decline in operations may have been due to concerns early in the pandemic about laparoscopy as an aerosol-generating procedure. The increased use of pre-operative testing of patients and measures to improve the COVID-safety of laparoscopy have allowed the careful re-starting of endometriosis surgery at some centres through 2020 and 2021, mitigating some of the disruption. However, a survey by the Royal College of Obstetricians and Gynaecologists in December 2020 found that only $20 \%$ of respondents felt that elective gynaecology activity at their units had returned to pre-2020 levels, indicating that the effect of the pandemic is likely to have extended well into 2021. 
The reduction in operations for severe endometriosis is unsurprising given the lack of operating theatre capacity and redeployment of staff. However, reduced patient access to GP services may be equally important, as well as other obstacles to obtaining a referral for endometriosis treatment, such as the need to self-isolate and reductions in endometriosis clinic capacity. In support of this, a report into elective care in England found that while there was a large decrease in the number of referred patients receiving definitive care in 2020 compared to 2019, there was an even larger decrease in the number of new referrals made, so that the total number of patients waiting for surgery actually decreased. As more patients are able to access GPs and obtain referrals to specialist endometriosis services, it is likely that most hospitals will experience a surge in referrals. Increased demand from primary care will add to those patients already awaiting surgery whose operations have been delayed, and existing services are unlikely to have capacity to meet the increasing demand. Furthermore, the ensuing delays in surgical treatment will cause additional costs to the NHS through emergency and pain management services, as well as other costs from lost time at work and education.

Considering the almost universal decline in centre activity, the BSGE has made a decision not to use operation numbers from 2020 as part of the accreditation process. This will allow many centres to remain on the BSGE centres list, and thereby ensure that the infrastructure is in place for an increase in operation numbers in the pandemic recovery period. Nevertheless, the effect on waiting times for endometriosis surgery will be dramatic, and the full extent of the impact on the wait for surgery is not yet known.

The sharp reduction in surgical case-load for highly complex surgery will undoubtedly have caused rippleeffects on proficiency, training and hands-on experience for both experienced and trainee-level endometriosis surgeons. "Buddy" operating, where experienced surgeons operate together, has been suggested by some to help reattain pre-pandemic levels of proficiency and confidence. This is a difficult debate as it represents a further cost pressure to organisations to fund what is regarded by some as a necessity. Meanwhile, new endometriosis surgeons will have felt the impact on their training acutely, with significant delays to the acquisition of new skills. Additional resources may therefore be required for advanced laparoscopic surgery training in order to maintain any benefit in additional surgical capacity for the longer term.

This study finds that reduction in endometriosis operations at a regional level are correlated with regional COVID-19 death rates. There are several reasons why this may be the case. Firstly, the repurposing of operating theatres for the care of COVID patients left few operating theatres available, particularly for elective surgery, and this effect may have been greater in regions with higher numbers of critically unwell COVID patients. Staffing required for endometriosis surgery is likely to have been worse affected by redeployment in regions worse affected by the pandemic, particularly affecting the availability of anaesthetists. Staff absence due to illness with COVID or a household member with COVID may have played a significant role. In fact, a survey by the Royal College of Physicians in April 2020 showed 21\% of staff away from work primarily due to these two reasons. Also, regions with worse COVID outbreaks may have seen fewer patients accessing GP services and endometriosis clinics due to a combination of reduced primary care services and patient avoidance of healthcare facilities ${ }^{15}$. Evidence that regions worse affected by the pandemic suffered greater reductions in endometriosis operations may be helpful in planning the allocation of resources so that operating capacity is increased where it is most needed. Indeed, greater sharing of patient pathways and working beyond single hospital boundaries may be required to smooth out some of the inequities in waiting times and access to care. However, this is only one of several factors to consider, and pre-pandemic waiting times will need to be taken into account alongside other concerns.

\section{Conclusion}

The effect of the COVID-19 pandemic on endometriosis surgery has been dramatic and should be addressed with urgency. A co-ordinated effort is required to establish increased capacity, wider access to care and greater support for patients and their clinical teams. This study puts the scale of the problem into focus, 
and it is hoped that this will encourage efforts to meet the challenge of promoting awareness and excellence in care for patients with endometriosis.

\section{Contributions to Authorship}

JL: primary author of paper including data aggregation and analysis, creation of figures, drafting and revision of text, corresponding author. ES: Editing of text, advising on methods and focus, revision of manuscript. DB: Editing of text, advising on methods and focus, revision of manuscript. JC: Editing of text, advising on methods and focus, revision of manuscript. AV: Conceptualisation of study, coordination of authors, editing of text, advising on methods and focus, revision of manuscript.

\section{Statement on Ethics}

This study did not involve any departure from routine clinical practice or divisions/randomisation of patients into groups. It was retrospective in nature, using pre-existing audits of national database data from the British Society for Gynaecological Endoscopy (BSGE). Full anonymity of patients was ensured through lack of any patient-specific data used, only the number of operations were known for each endometriosis centre per year. For these reasons prior approval from a research ethics committee was not required. The audits were registered annually and performed by the BSGE, and are publicly available on their website. Approval was gained from the BSGE for the study.

\section{Funding}

No funding was provided or required for the study.

\section{Discolsure of Interests}

Professor Thomas Justin Clark is the current president of the British Society for Gynaecological Endoscopy. There are no other relevant financial, personal, political, intellectual or other interests to declare.

\section{Figure List}

Figure 1: Number of BSGE registered operations and centres in each year.

Figure 2: Boxplot of number of BSGE registered operations in each year at each endometriosis centre. Each datapoint represents data from one centre in each year.

Figure 3: Graph showing correlation between the percentage reduction in operations for endometriosis (compared to 2017-2019 mean) with COVID deaths per 1000 of population. Linear regression line and $95 \%$ confidence interval band shown.

Figure 4: Geographic plot of reduction in operations compared to previous 3-year average (left) and COVID deaths per 1000 population.

References 


\section{Acknowledgements}

The authors would like to thank Conor Byrne and team at the ICEway IT services for management of the database and data download used in this study.

\section{Hosted file}

Figure1.docx available at https://authorea.com/users/437476/articles/539084-impact-of-thecovid-19-pandemic-on-surgery-for-severe-endometriosis-in-the-uk-a-national-databasestudy

\section{Hosted file}

Figure 2.docx available at https://authorea.com/users/437476/articles/539084-impact-of-thecovid-19-pandemic-on-surgery-for-severe-endometriosis-in-the-uk-a-national-databasestudy

\section{Hosted file}

Figure 3.docx available at https://authorea.com/users/437476/articles/539084-impact-of-thecovid-19-pandemic-on-surgery-for-severe-endometriosis-in-the-uk-a-national-databasestudy

\section{Hosted file}

Figure 4.docx available at https://authorea.com/users/437476/articles/539084-impact-of-thecovid-19-pandemic-on-surgery-for-severe-endometriosis-in-the-uk-a-national-databasestudy 